\title{
BRUCE silencing leads to axonal dystrophy by repressing autophagosome-lysosome fusion in Alzheimer's disease
}

\author{
Lu Zhang $\mathbb{D}^{1}$, Yu Fang ${ }^{2}$, Xinyu Zhao', Yake Zheng ${ }^{1}$, Yunqing Ma', Shuang Li ${ }^{1}$, Zhi Huang ${ }^{1}$ and Lihao Li ${ }^{1}$
}

\begin{abstract}
Axonal dystrophy is a swollen and tortuous neuronal process that contributes to synaptic alterations occurring in Alzheimer's disease (AD). Previous study identified that brain-derived neurotrophic factor (BDNF) binds to tropomyosin-related kinase B (TrkB) at the axon terminal and then the signal is propagated along the axon to the cell body and affects neuronal function through retrograde transport. Therefore, this study was designed to identify a microRNA (miRNA) that alters related components of the transport machinery to affect BDNF retrograde signaling deficits in AD. Hippocampus tissues were isolated from APP/PS1 transgenic (AD-model) mice and C57BL/6J wild-type mice and subject to nicotinamide adenine dinucleotide phosphate and immunohistochemical staining.

Autophagosome-lysosome fusion and nuclear translocation of BDNF was detected using immunofluorescence in HT22 cells. The interaction among miR-204, BIR repeat containing ubiquitin-conjugating enzyme (BRUCE) and Syntaxin 17 (STX17) was investigated using dual luciferase reporter gene assay and co-immunoprecipitation assay. The expression of relevant genes and proteins were determined by RT-qPCR and Western blot analysis. Knockdown of STX17 or BRUCE inhibited autophagosome-lysosome fusion and impacted axon growth in HT22 cells. STX17 immunoprecipitating with BRUCE and co-localization of them demonstrated BRUCE interacted with STX17. BRUCE was the target of miR-204, and partial loss of miR-204 by inhibitor promoted autophagosome-lysosome fusion to prevent axon dystrophy and accumulated BDNF nuclear translocation to rescue BDNF/TrkB signaling deficits in HT22 cells. The overall results demonstrated that inhibition of miR-204 prevents axonal dystrophy by blocking BRUCE interaction with STX17, which unraveled potential novel therapeutic targets for delaying AD.
\end{abstract}

\section{Introduction}

Dystrophic axons (axonal dystrophy), also known as dystrophic neuritis or neuroaxonal dystrophy, are considered one of the major neuropathological features of Alzheimer's disease $(\mathrm{AD})^{1}$. It has been revealed that dystrophic axons contained the accumulated tubulovesicular structures, filamentous materials, and swollen edematous mitochondria with a degenerated inner membrane that are localized in the central nervous system ${ }^{2}$. The two

\footnotetext{
Correspondence: Lu Zhang (zhangluzl18@163.com)

'Department of Neurology, The First Affiliated Hospital of Zhengzhou University, Zhengzhou 450052, P.R. China

${ }^{2}$ ICU, The First Affiliated Hospital of Zhengzhou University, Zhengzhou 450052, P.R. China
}

major diseases of dystrophic axons are pantothenate kinase-associated neurodegeneration and infantile neuroaxonal dystrophy ${ }^{3}$. Previous studies have reported that phosphodiesterase inhibitors can reverse axonal dystrophy in mice with Friedreich's ataxia, and a brain-penetrant triazolopyrimidine alleviates the axonal dysfunction in tauopathy mice ${ }^{4,5}$. Nevertheless, the exact mechanism of the dystrophic axons in AD remains to be elucidated.

Interestingly, it has been revealed that the neurotrophins function as a crucial regulator of axon regeneration, especially brain-derived neurotrophic factor $(B D N F)^{6}$. Neurotrophins are endogenous peptides that enhance the survival and maintenance of neurons. The member of neurotrophins, BDNF, has been revealed to

\section{(c) The Author(s) 2021}

(c) (i) Open Access This article is licensed under a Creative Commons Attribution 4.0 International License, which permits use, sharing, adaptation, distribution and reproduction cc) in any medium or format, as long as you give appropriate credit to the original author(s) and the source, provide a link to the Creative Commons license, and indicate if changes were made. The images or other third party material in this article are included in the article's Creative Commons license, unless indicated otherwise in a credit line to the material. If material is not included in the article's Creative Commons license and your intended use is not permitted by statutory regulation or exceeds the permitted use, you will need to obtain permission directly from the copyright holder. To view a copy of this license, visit http://creativecommons.org/licenses/by/4.0/. 
exert neuroprotective effects in different animal models of neurodegeneration ${ }^{7}$. In addition, previous researches have reported that retrograde transport of BDNF-activated tropomyosin-related kinase B (TrkB) receptors was involved in the process of autophagosomes, which improves the neuronal complexity and preventing neurodegeneration in vivo, and the BDNF expression was modulated by sortilin-mediated trafficking and lysosomal degradation $^{8,9}$. Lysosomes have been found to be significant in maintaining the integrity of neuronal function, and mutations in genes inducing lysosome formation, transport, and activity related to neurodegenerative disorders $^{10}$. In addition, the inhibitor of apoptosis family of proteins (IAP) BIR repeat-containing ubiquitin-conjugating enzyme (BRUCE) was reported to modulate the fusion of autophagosome and lysosome, and also interacted with Syntaxin 17 (STX17), an important mediator in autophagosome-lysosome fusion ${ }^{11}$. The function of STX17 as a crucial protein during the fusion of autophagosome and lysosome in non-cardiomyocytes has also been demonstrated previously ${ }^{12}$. Furthermore, the upregulation of microRNA-204 (miR-204) in the distal axons of sympathetic neurons has been identified ${ }^{13}$ and was involved in the regulation of transcription and informatics-identified pathways in the processes of neurogenesis and axon guidance ${ }^{14}$. Interestingly, miR-204 has been proved to target BDNF in tumor progression ${ }^{15}$. Therefore, from the findings above, it was hypothesized that the miR-204/BRUCE/STX17/BDNF axis may affect the development of dystrophic axons in $\mathrm{AD}$ by regulating the fusion of autophagosome and lysosome. Hence, the present study aimed to test this hypothesis and explore the underlying mechanism of axonal dystrophy in $\mathrm{AD}$, which provides insight into the therapeutic targets for the treatment for $\mathrm{AD}$.

\section{Methods and materials Ethics statement}

All of the experiments involving rodents in this study were approved by the Committee on Animal Care and Use from the First Affiliated Hospital of Zhengzhou University. All animal experiments were performed in strict accordance with the recommendations in the Guide for the Care and Use of Laboratory Animals of the National Institutes of Health and conform to relevant national provisions.

\section{Study subjects}

The male $\mathrm{APP}_{\mathrm{SWE}} / \mathrm{PS}_{\triangle \mathrm{E} 9}$ transgenic mice (age: 12 weeks) and C57BL/6J wild-type (Wt) mice (age: 12 weeks) that used in this study were purchased from HFK Biotechnology Co., Ltd. (Beijing, China). The mice were reared in the cage with polypropylene $(34 \times 22 \times$ $15 \mathrm{~cm}$ ) under standard conditions (room temperature of
$22.5 \pm 1{ }^{\circ} \mathrm{C}$, humidity of $50 \pm 2 \%$ and $12 \mathrm{~h}$ of light and dark cycle) with free access to food and water. For tissue analysis, all mice were euthanized by $\mathrm{CO}_{2}$ asphyxiation, and the hippocampus was isolated from the mice brain, sectioned into $4 \mu \mathrm{m}$, and stained by nicotinamide adenine dinucleotide phosphate (NADPH-d). Subsequently, the sections were observed and analyzed under a microscope.

The procedures of NADPH-d staining were as follows: frozen sections were pre-incubated for $5-10 \mathrm{~min}$ in Trisbuffer solution containing Triton X-100, and incubated for $1-1.5 \mathrm{~h}$ in Tris-buffer solution containing Triton X100, NADPH-d, and nitroblue tetrazolium. After the termination of the reaction, the sections were cleared and mounted.

\section{Immunohistochemistry (IHC)}

Sectioned hippocampus tissues were incubated with diluted mouse anti-BDNF antibody $(5 \mu \mathrm{g} / \mathrm{mL}$, ab203573, Abcam, Cambridge, UK) for $16 \mathrm{~h}$ at $4{ }^{\circ} \mathrm{C}$ and with horseradish peroxidase (HRP)-labeled rabbit anti-mouse immunoglobulin G (IgG) (ab6728, Abcam) for $2 \mathrm{~h}$. Subsequently, the sections were stained for $5 \mathrm{~min}$ with diaminobenzidine (AR1000; Wuhan Boster Biological Technology Co., Ltd., Wuhan, Hubei, China), then observed and photographed under the optical microscope.

\section{Harvest of primary hippocampal neurons HT22}

In brief, primary culture was obtained from mouse hippocampus on the 18th embryonic day, dissected under sterile condition, and detached with $0.125 \%$ trypsin at $37^{\circ} \mathrm{C}$ for $20 \mathrm{~min}$. Then, complete medium (Neurobasal2, Gibco, Grand Island, NY, USA) containing 2\% B27 (Gibco), $2 \mathrm{mM}$ alanyl-glutamine (Gibco), 10\% fetal bovine serum (FBS), and $1 \mathrm{mM}$ penicillin/streptomycin (Sigma, St. Louis, MO, USA) were applied to terminate the detachment. Tissues were washed by FBS-free complete medium and dissociated using a plastic pipette. Neurons were spread on a petri dish with a glass bottom at a density of $0.25-1 \times 10^{5}$ cells $/ \mathrm{mL}$ and allowed to stand for $2 \mathrm{~h}$ in the incubator for attachment. Then, a serum-free medium was added until the volume reached $2 / 3$. Primary hippocampal neurons HT22 (CL-0595; Procell Life Science \& Technology Co., Ltd., Hubei, China; https://www. procell.com.cn/view/4535.html) were used for in vitro experiments in this study. HT22 cells were cultured in the Dulbecco's modified Eagle's medium containing 10\% FBS, 2 mM L-alanyl-L-glutamate, 0.24\% hydroxyethyl piperazine ethane-sulfonic acid (HEPES), $0.375 \%$ sodium bicarbonate, $100 \mathrm{U} / \mathrm{mL}$ penicillin, and $100 \mathrm{mg} / \mathrm{mL}$ streptomycin.

\section{Cell treatment}

HT22 cells were detached with trypsin, counted, seeded in a 6 -well plate at a density of $5 \times 10^{5}$ cells/well, and 
allowed to stand overnight for attachment. Upon reaching $80 \%$ confluence, subsequent experiments were performed. HT22 cells were washed 3 times with Dulbecco's phosphate-buffered saline (DBPS) and cultured in starvation medium ( $40 \mathrm{mM} \mathrm{NaCl}, 1 \mathrm{mM} \mathrm{CaCl}, 1 \mathrm{mM} \mathrm{MgCl}_{2}$, $5 \mathrm{mM}$ glucose, and $20 \mathrm{mM}$ HEPES, $\mathrm{pH} 7.4$ ) at $37^{\circ} \mathrm{C}$ in order to allow the cells to achieve a starvation state.

To block the autophagy flux, part of the cells were interfered with using Bafilomycin A1 (Abmole Bioscience Inc., CA, USA) at concentrations of 10,20 , and $50 \mathrm{nM}$, respectively. The autophagosome staining was performed using an MDC kit (P6659-20 $\mu \mathrm{g}$ Recombinant Human MDC/CCL22 $20 \mu \mathrm{g}$, Beyotime Biotechnology Co., Ltd., Shanghai, China), and lysosome staining was performed using Lys-tracker-Red kit [C1046 Lyso-Tracker Red (lysosome red fluorescent probe) $50 \mu \mathrm{L}$, Beyotime]. After $24 \mathrm{~h}$ of treatment, the cells were photographed and observed under microscope ${ }^{16}$.

For cell transfection, the adenoviruses pAd/CMV/V5DEST and pAd/BLOCK-iT-DEST RNAi Gateway Vector were purchased from BioVector NTCC (Beijing, China). The overexpression of BRUCE (oe-BRUCE) and short hairpin RNA against STX17 (sh-STX17) and BRUCE (shBRUCE), as well as corresponding negative control (NC) (oe-NC and sh-NC), were purchased from GenePharma Co., Ltd. (Shanghai, China). Also, the miR-204 mimic/ inhibitor was purchased from GenePharma. The cell transfection was conducted in accordance with the manufacturer's instructions of Lipofectamine 2000 (Invitrogen, Carlsbad, CA, USA). In addition, the construction of HT22 cell line with dominant-negative STX17 mutant (Mut) GFP-STX17 $\triangle$ NTD or full-length STX17 GFPSTX17FL overexpression was performed based on a previous study ${ }^{12}$.

\section{Western blot analysis}

HT22 cells or mouse hippocampus tissues were lysed for total protein extraction. For nuclear protein extraction, HT22 cells or mouse hippocampus tissues were lysed and centrifuged at $4000 \mathrm{rpm}$ to collect the precipitate. Then, the precipitate was resuspended and centrifuged at $13,000 \mathrm{rpm}$ for $7 \mathrm{~min}$. Total or nuclear protein $(40 \mu \mathrm{g}$ of protein per lane) was separated by $10 \%$ sodium dodecyl sulfate (SDS)-polyacrylamide gel electrophoresis and transferred onto the polyvinylidene fluoride membrane (Millipore, Billerica, MA, USA). Subsequently, the membrane was blocked with 5\% skimmed milk powder at room temperature for $2 \mathrm{~h}$, and then incubated at $4{ }^{\circ} \mathrm{C}$ overnight with following primary antibodies: rabbit anti-BDNF (1:1000, ab108319), phosphorylated TrkB (p-TrkB)/TrkB (1:500, 4621/4606), rabbit anti-p-cAMPresponse element-binding protein (CREB) (1:50, ab32096), rabbit anti-CREB (1:1000, ab32515), rabbit antiSTX17 (1:1000, ab229646), and rabbit anti-BRUCE
(1:5000, ab19609). All the antibodies above were purchased from Abcam except p-TrkB/TrkB (Cell Signaling Technology, Beverly, MA, USA). After overnight incubation, the membrane was incubated with secondary antibody HRP-labeled anti-rabbit IgG (1:20,000, Thermo Fisher Scientific, MA, USA). Glyceraldehyde-3-phosphate dehydrogenase (GAPDH) or LaminA served as an internal reference, and the protein bands were observed using an electrochemiluminescence kit (CoWin Biosciences, Beijing, China).

\section{Reverse transcription-quantitative polymerase chain reaction (RT-qPCR)}

The total RNA was extracted from mouse hippocampus tissues or HT22 cells. Reverse transcription was done according to the manufacturer's instructions of SuperScript III First-Strand Synthesis SuperMix. Then, the expression of miR-204 was detected using TaqMan miRNA assay (Ambion, Austin, TX, USA) with U6 used as the internal reference, while the mRNA expression of other genes was examined using PrimeScript RT-PCR kits (Roche, Basel, Switzerland) with GAPDH used as the internal reference. All primers were synthesized by Takara Biotechnology Ltd. (Dalian, Liaoning, China) (Table S1). The fold changes of target genes between the experimental group and the control group were calculated using the $2^{-\Delta \Delta \mathrm{Ct}}$ method.

\section{Immunofluorescence staining}

The cells grown on slides were fixed with $4 \%$ paraformaldehyde for $15 \mathrm{~min}$ and blocked with $1 \times$ phosphate buffer saline supplemented with $3 \mathrm{mg} / \mathrm{mL}$ bovine serum albumin, $100 \mathrm{mM}$ glycine, and $0.25 \%$ Triton X-100 for $30 \mathrm{~min}$. Subsequently, the slides were incubated with primary antibody rabbit anti-BDNF (1:500, ab108319), rabbit anti-light chain 3II (LC3II) (1:1000, ab48394), mouse anti-Tau (Tau-13, 1:500, Santa Cruz Biotechnology, Santa Cruz, CA, USA) and rabbit anti-BRUCE $(5 \mu \mathrm{g} /$ $\mathrm{mL}$, ab19609) at $4{ }^{\circ} \mathrm{C}$, followed by culture with the combination of the fluorophore and Alexa Fluor ${ }^{\circledR} 647 \mathrm{sec}-$ ondary antibody (1:200, ab150075) at room temperature for $1 \mathrm{~h}$. All the antibodies used above were provided by Abcam except Tau. Then, the cell slides were added with 4' 6-diamidino-2-phenylindole for nucleus staining, then immersed in distilled water, dried and observed under a fluorescence microscope (Zeiss, Thornwood, NY) or FV1000 confocal microscope.

For morphological analysis, the cells were fixed with $4 \%$ paraformaldehyde for $15 \mathrm{~min}$ and incubated with tau antibody (1:200, ab64193, Abcam) for $1 \mathrm{~h}$, followed by another incubation with fluorophore combined with Alexa Fluor ${ }^{\circledR} 647$ secondary antibody (1:200, ab150075) for $1 \mathrm{~h}$. The cells were then observed under the fluorescence microscope, and the length of the main 
axon in each cell was measured by five independent experiments.

\section{Dual-luciferase reporter gene assay}

The synthetic BRUCE-3' untranslated region (UTR) gene fragments were introduced into pMIR-reporter (Beijing Huayueyang Biotechnology Co., Ltd., Beijing, China) using endonucleases SpeI and Hind III. With the complementary sequence Mut site of seed sequence designed in BRUCE Wt, the targeted fragments after restriction endonuclease digestion were inserted into the pMIR-reporter plasmid.

The correctly sequenced luciferase reporter plasmids, $\mathrm{Wt}$ and Mut were co-transfected with miR-204 and transfected into HEK-293T cells (CRL-1415, Shanghai Xin Yu Biotech Co., Ltd., Shanghai, China). After $48 \mathrm{~h}$, the cells were collected and lysed. Lastly, the luciferase activity was determined using the Luciferase Detection Kit (RG005, Beyotime) on Glomax20/20 Luminometer Fluorescence Detector (Promega, Madison, WI, USA).

\section{Co-immunoprecipitation (Co-IP) assay}

HT22 cell lysates were collected, and one part was used as input. Every $500 \mu \mathrm{g}$ of lysate was incubated with antibody and Dynabeads Protein G (Invitrogen, Carlsbad, CA, USA) at $4{ }^{\circ} \mathrm{C}$ for protein complexes to pull down. The IgG (Santa Cruz Biotechnology, Inc., Santa Cruz, CA, USA) served as the NC. Subsequently, the beads were washed with cold lysis buffer and boiled in 1× SDS loading buffer for $15 \mathrm{~min}$ to elute proteins, followed by Western blot analysis.

\section{Statistical analysis}

All data were statistically analyzed using SPSS 21.0 statistical software (IBM Corp., Armonk, NY, USA). Measurement data were expressed as mean \pm standard deviation. Data following normal distribution and homogeneity of variance were compared by unpaired $t$-test between two groups. Data among multiple groups were compared by one-way analysis of variance followed by Tukey's post hoc test. The repeated-measures ANOVA was applied for data among multiple groups at different time points, followed by the Bonferroni post hoc test. $p<0.05$ was considered as statistically significant.

\section{Results}

\section{BDNF/TrkB signaling is implicated in AD mice}

The length and morphological structure of axons in the hippocampus of $\mathrm{APP}_{\mathrm{SWE}} / \mathrm{PS}_{\triangle \mathrm{E} 9}$ transgenic $\mathrm{AD}$ mice and C57BL/6J Wt mice were analyzed, as shown in Fig. 1A. Compared with the Wt mice, AD mice exhibited shorter and swollen axons. Then, IHC was conducted to examine the BDNF expression in the hippocampus tissues. Results revealed that the BDNF expression in the nucleus was sharply decreased in the hippocampus tissues of $\mathrm{AD}$ mice relative to Wt mice (Fig. 1B). Meanwhile, it was further proved by Western blot analysis that BDNF expression in

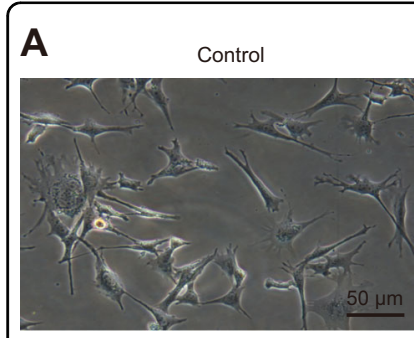

C

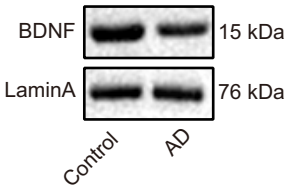

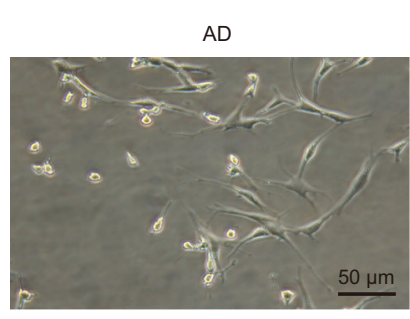

D
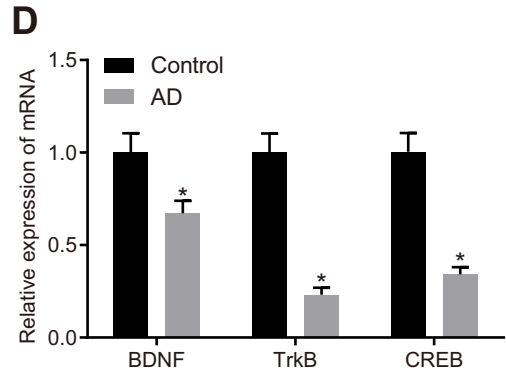

B Control
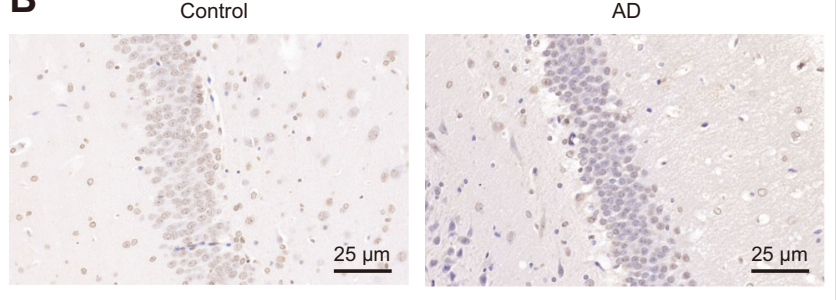

E

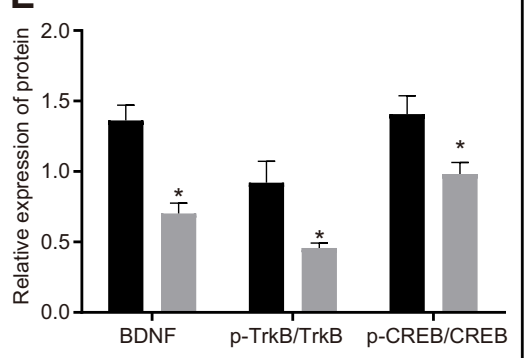

Fig. 1 BDNF/TrkB signaling is associated with dystrophic axons in APP/PS1 transgenic mice. The mice used for the following detection were $\mathrm{APP}_{S W E} / P S 1_{\triangle E 9}$ transgenic AD mice $(n=6)$ and $\mathrm{C57BL} / 6 \mathrm{~J}$ Wt mice $(n=6)$. A The length and morphological structure of axons in the hippocampus of AD mice and Wt mice observed under an optical microscope (200X). B The intranuclear BDNF expression in the hippocampus tissues of mice examined by IHC (400×). C The expression of BDNF in the nucleus of the hippocampus tissues of mice detected by Western blot analysis, ${ }^{*} p<0.05$ compared with that of Wt mice. D The mRNA expression of BDNF, TrkB, and CREB in the hippocampus tissues of AD mice and Wt mice detected by RT-qPCR, ${ }^{*} p<0.05$ compared with that of Wt mice. $\mathbf{E}$ The protein expression of BDNF, $p$-TrkB/TrkB, and p-CREB/CREB in the hippocampus tissues of $\mathrm{AD}$ mice and Wt mice examined by Western blot analysis, ${ }^{*} p<0.05$ compared with that of Wt mice. The experiment was repeated three times independently. 
the nucleus was markedly lower in $\mathrm{AD}$ mice when compared to Wt mice (Fig. 1C). In addition, RT-qPCR and Western blot analyses were performed to detect the mRNA and protein expression of BDNF/TrkB signaling pathway-related factors (BDNF, p-TrkB/TrkB, and pCREB/CREB). Results demonstrated that the mRNA and protein expression of BDNF, $p-\operatorname{Trk} B / \operatorname{TrkB}$, and p-CREB/ CREB was notably reduced in the hippocampus tissues of AD mice when compared to Wt mice (Fig. 1D, E). Hence, these finding indicates that BDNF/TrkB signaling is affected in AD mice.

\section{Autophagosome-lysosome fusion mediates BDNF nuclear transport in HT22 cells}

In order to further explore the mechanism underlying nuclear transport of BDNF, HT22 cells were initially treated with starvation culture and then added with proton pump inhibitor Bafilomycin A1 to inhibit the fusion of autophagosome and lysosome ${ }^{17}$. The cells were interfered with using Bafilomycin A1 at concentrations of 10, 20, and $50 \mathrm{nM}$, respectively for $24 \mathrm{~h}$, the cells were then photographed, recorded and immunofluorescence staining was performed subsequently. Results demonstrated that the autophagosome-lysosome fusion reduced with the increasing concentration of Bafilomycin $\mathrm{A} 1$, and the autophagosome-lysosome fusion was inhibited most dramatically after the interference using $50 \mathrm{nM}$ Bafilomycin $\mathrm{A} 1$, as presented in Fig. 2A. Also, the BDNF signaling nuclear import was gradually decreased with the increasing concentration of Bafilomycin A1, and the significant changes of BDNF signaling nuclear import were detected after treatment of $50 \mathrm{nM}$ Bafilomycin A1 (Fig. 2B). In addition, results from Western blot analysis showed that when compared with cells without treatment, the cells treated with Bafilomycin A1 presented an obvious decrease in protein expression of $\mathrm{BDNF} / \mathrm{TrkB}$ signaling pathwayrelated factors and BDNF protein expression in the nucleus (Fig. 2C, D). After 24-h treatment with Bafilomycin A1, the axon length and cell body of HT22 cells were obviously reduced upon treatment of $30 \mathrm{nM}$ Bafilomycin A1 (Fig. 2E, $\mathrm{F}$ and Fig. S1A). In a word, autophagosomes-lysosomes fusion regulated BDNF nuclear transport and further led to axonal dystrophy.

\section{Knockdown of STX17 inhibits the autophagosome-lysosome fusion and affects the axon growth in HT22 cells}

To investigate the mechanism underlying autophagosome-lysosome fusion, the HT22 cell line with GFP-STX17 $\triangle$ NTD or GFP-STX17FL overexpression was constructed, followed by conduction of immunofluorescence staining. The results revealed that when compared with cells without treatment, the GFP-STX17 $\triangle$ NTD-HT22 cells presented remarkably increased LC3II and STX17 expression and colocalization of LC3II and STX17. On the other hand, the GFP-STX17FL-HT22 cells showed no obvious change in LC3II expression but significantly increased STX17 (Fig. 3A). HT22 cell line with silenced STX17

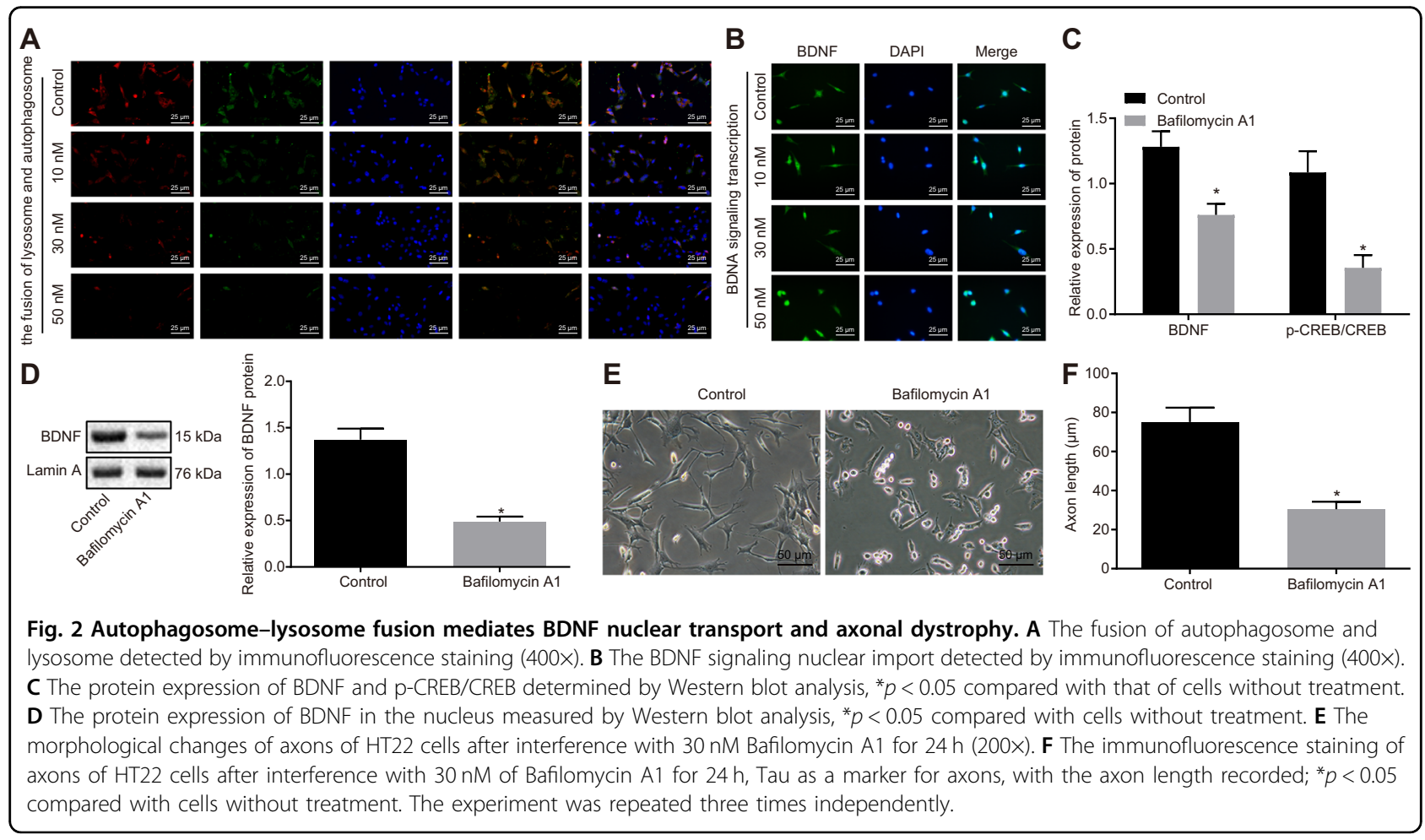




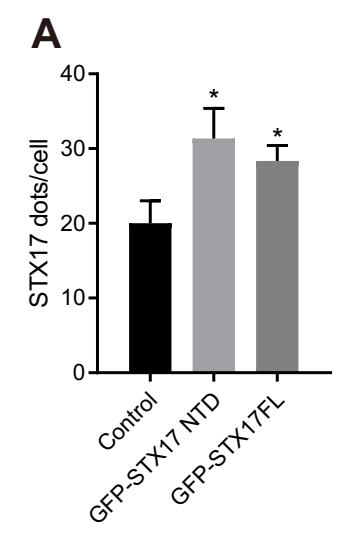

C

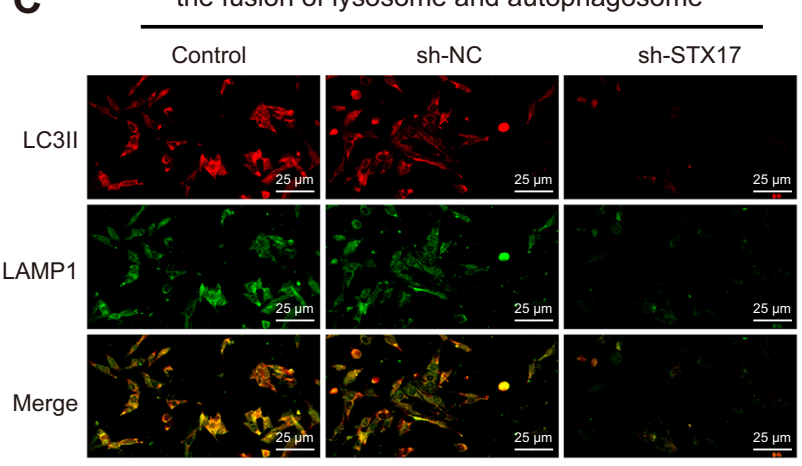

B

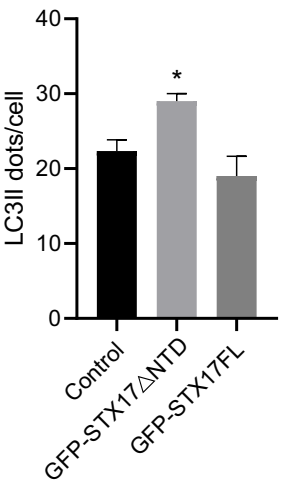

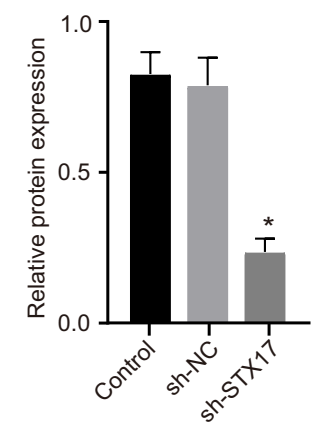
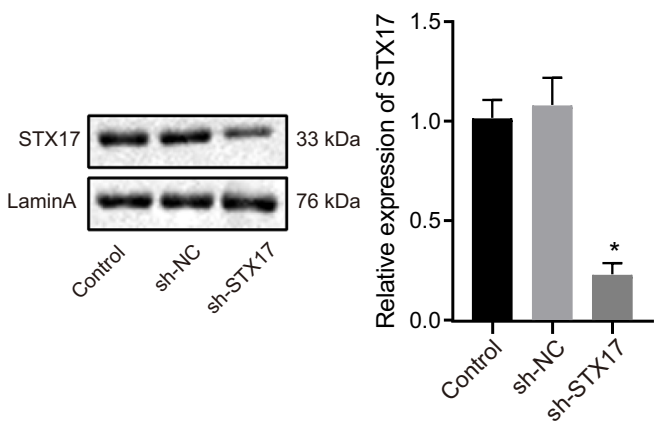

D

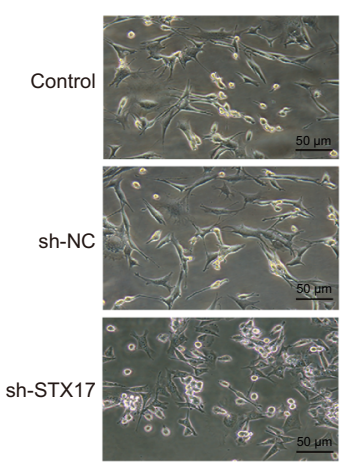

E

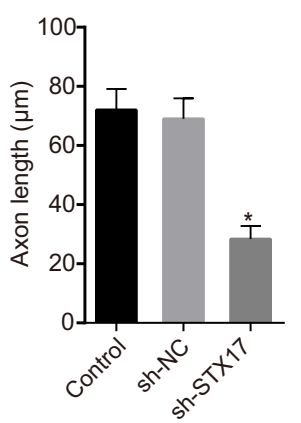

Fig. 3 STX17 knockdown inhibits the autophagosome-lysosome fusion and reduces axon growth in HT22 cells. A Co-localization of LC3॥ and STX17 in HT22 cells without treatment, GFP-STX17 $\triangle$ NTD-HT22 cells, or GFP-STX17FL-HT22 cells determined by immunofluorescence staining, ${ }^{*} p<0.05$ compared with cells without treatment. The HT22 cells were treated with sh-NC or sh-STX17. B The silencing efficiency of STX17 determined by RT-qPCR and Western blot analysis, ${ }^{*} p<0.05$ compared with cells treated by sh-NC. $\mathbf{C}$ The fusion of autophagosome and lysosome in cells (400x). D The morphological changes of axons in cells (200x). E The immunofluorescence staining of axons in cells detected by immunofluorescence staining, ${ }^{*} p<0.05$ compared with cells treated by sh-NC. The experiment was repeated three times independently.

was constructed, and the silencing efficiency was confirmed by RT-qPCR and Western blot analysis (Fig. 3B). Subsequently, the fusion of autophagosome and lysosome was determined. In comparison with sh-NCtreated cells, the autophagosome-lysosome fusion in sh-STX17-treated cells showed a marked decrease (Fig. 3C). Based on the morphology of axons in HT22 cells, it was found that the axon and cell body had no pronounced change in cells treated with sh-NC but the axon length and size of cell body were decreased in cells treated with sh-STX17 in relative to the cells without treatment (Fig. 3D, E and Fig. S1B). In short, STX17 silencing is able to reduce the fusion of autophagosomes and lysosomes, and may thus further affect the growth of axons.

\section{Knockdown of BRUCE suppresses the} autophagosome-lysosome fusion by interacting with STX17

The Co-IP assay for exploring the relationship between BRUCE and STX17 revealed an interaction between BRUCE and STX17 (Fig. 4A). The immunofluorescence staining further verified that the existence of colocalization between BRUCE and STX17 (Fig. 4B). These results demonstrated that BRUCE might interact with STX17 and regulate the fusion of autophagosome and lysosome. To validate this hypothesis, HT22 cells were treated with silenced BRUCE, followed by Western blot analysis. Results revealed that HT22 cells exhibited notably decreased BRUCE protein expression, confirming the successful silencing (Fig. 4C). Immunofluorescence staining was performed to detect the fusion of autophagosome and lysosome, and it was found that when compared with cells without treatment, cells treated with sh-NC showed no marked change in autophagosome-lysosome fusion. In comparison with cells treated with sh-NC, the cells treated with shBRUCE reduced autophagosome-lysosome fusion (Fig. 4D) as well as decreased BDNF nuclear import (Fig. 4E). In addition, the results from Western blot analysis revealed that when compared with the cells treated with sh-NC, the protein expression of BDNF/ TrkB signaling pathway-related factors and BDNF 


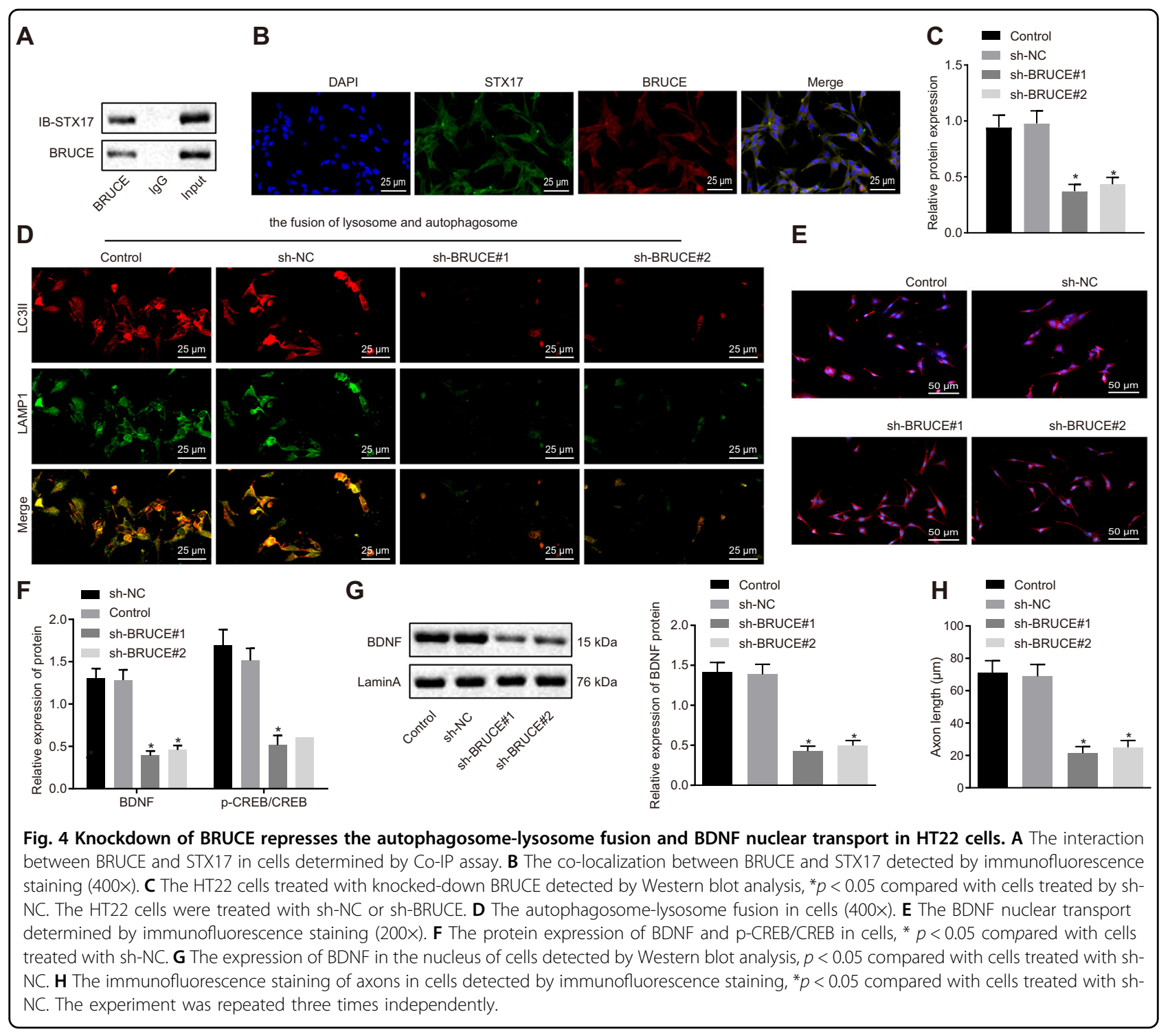

protein expression in the nucleus were dramatically decreased in cells transfected with sh-BRUCE (Fig. 4F, G). Also, the axon length and size of the cell body were also reduced in cells treated with sh-BRUCE relative to cells treated with sh-NC (Fig. $4 \mathrm{H}$ and Fig. S1C). Therefore, the interaction between BRUCE and STX17 can affect the nuclear transport of BDNF, thereby regulating the fusion of autophagosomes and lysosomes.

\section{miR-204 causes axonal dystrophy in AD by decreasing BRUCE}

Furthermore, RT-qPCR was conducted to detect the expression of miR-204 in the hippocampus tissues of mice. Results revealed that miR-204 was obviously upregulated in the hippocampus tissues in $\mathrm{AD}$ mice when compared with normal mice (Fig. 5A). In silico analysis revealed that there was a binding site between miR-204 and BRUCE 3'UTR (Fig. 5B), and dual-luciferase reporter gene assay further identified that miR-204 could effectively inhibit BRUCE (Fig. 5C). HT22 cells were transfected with miR-204-mimic/inhibitor and their controls, followed by RT-qPCR and Western blot analysis to examine the mRNA and protein expression of BRUCE. In comparison with cells treated with mimic-NC, cells treated with miR-204 mimic showed significantly reduced mRNA and protein expression of BRUCE, while the cells treated with miR-204 inhibitor exhibited obviously increased mRNA and protein expression of BRUCE in relative to cells treated with inhibitor-NC (Fig. 5D, E). The immunofluorescence staining was conducted to detect the fusion of autophagosome and lysosome. Results revealed that the autophagosome-lysosome fusion was reduced in cells treated with miR-204 mimic but obviously enhanced in cells treated with miR-204 inhibitor (Fig. 5F). Next, 


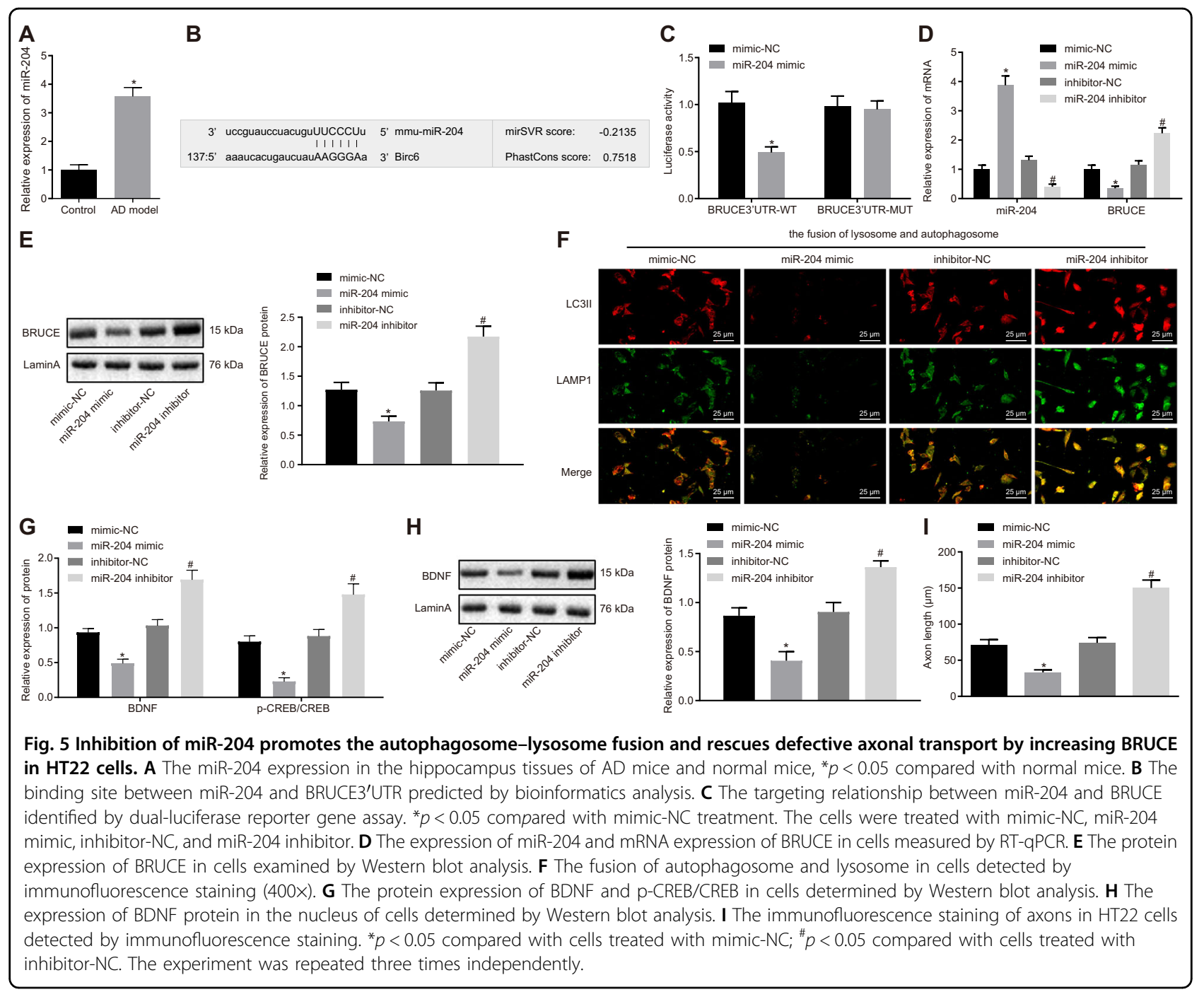

results from the Western blot analysis showed that miR204 mimic treatment resulted in a significant reduction in protein expression of $\mathrm{BCNF} / \mathrm{TrkB}$ signaling pathwayrelated factors while miR-204 inhibitor treatment showed an opposite result (Fig. 5G, H). Based on the morphology of axons in HT22 cells, it was found that the axon length and size of the cell body were markedly decreased in cells treated with miR-204 but increased in cells treated with miR-204 inhibitor (Fig. 5I and Fig. S1D). In a nutshell, inhibition of BRUCE by miR-204 reduces the fusion of autophagosomes and lysosomes, which further leads to axonal dystrophy in AD mice.

\section{Overexpression of BRUCE rescues miR-204-triggered axonal dystrophy in HT22 cells}

In order to verify the effects of miR-204 and BRUCE in dystrophic axons of HT22 cells, experiments with overexpressed BRUCE were conducted. The results of RTqPCR revealed that compared with cells treated with both
mimic-NC and oe-NC, cells co-treated with miR-204 mimic and oe-NC showed a marked increase in miR-204 expression but an obvious decrease in mRNA expression of BRUCE was observed. In comparison with cells cotreated with miR-204 mimic and oe-NC, cells with combined treatment of miR-204 mimic and oe-BRUCE showed no pronounced change in miR-204 expression but showed an obvious increase in mRNA expression of BRUCE (Fig. 6A). Meanwhile, Western blot analysis was conducted to examine the protein expression of BRUCE in cells. It was found that protein expression of BRUCE was notably reduced when combined treated with miR204 mimic and oe-NC but was obviously elevated after cotreated with miR-204 mimic and oe-BRUCE (Fig. 6B). Subsequently, immunofluorescence staining was performed to detect the fusion of autophagosome and lysosome. Results revealed that the combined treatment of miR-204 mimic and oe-NC resulted in markedly repressed autophagosome-lysosome fusion, while co-treatment 

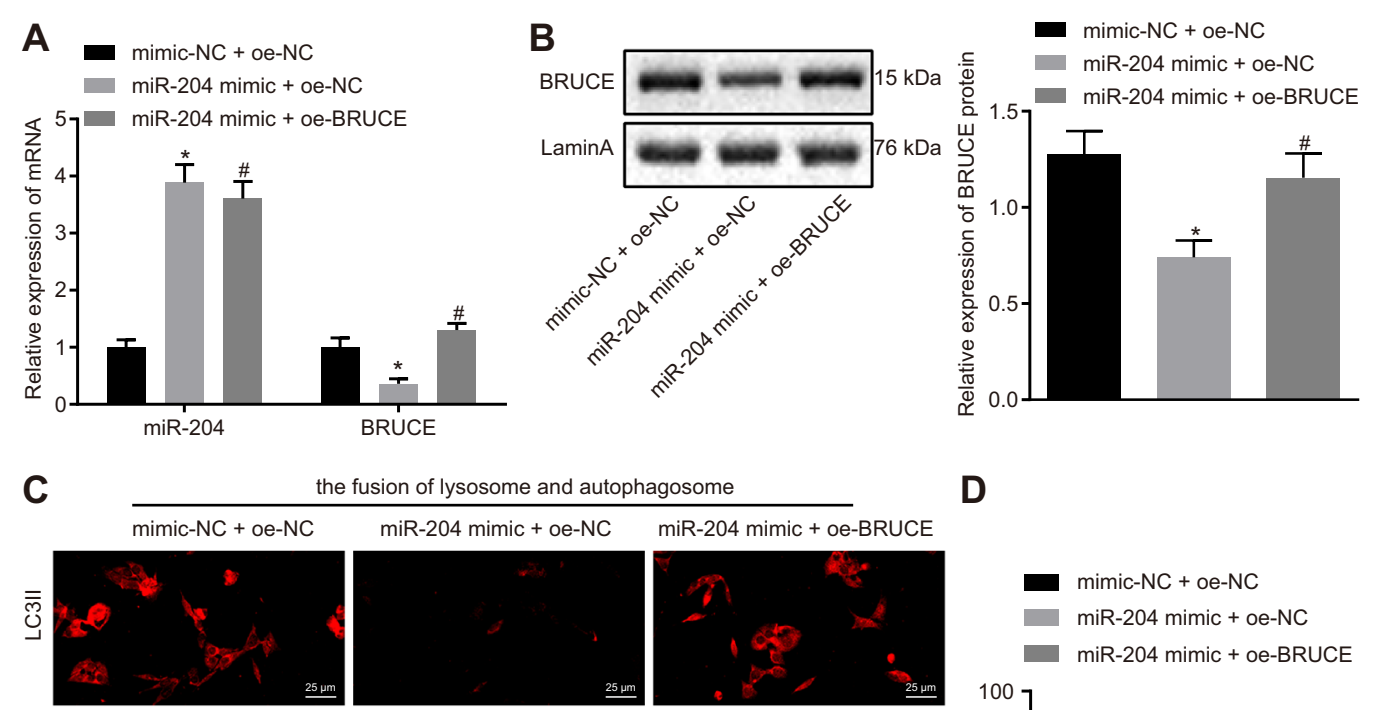

the fusion of lysosome and autophagosome
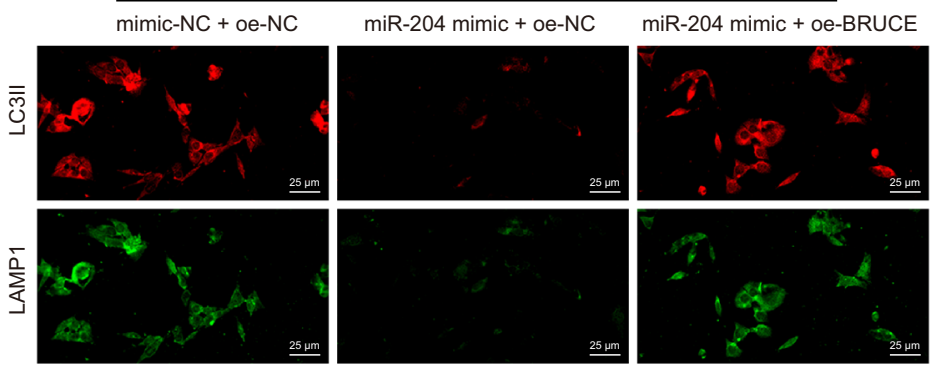

D
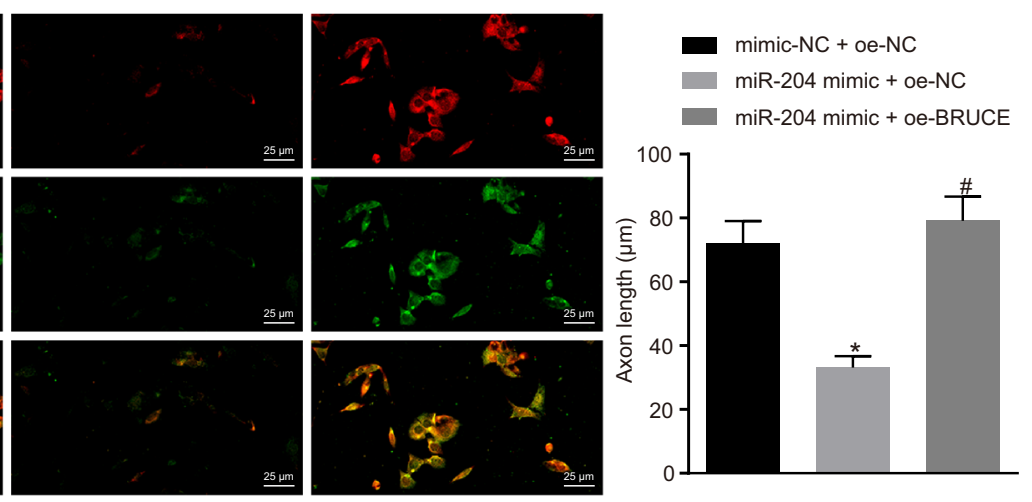

Fig. 6 Overexpression of BRUCE rescues axons in HT22 cells. The HT22 cells were treated with both mimic-NC and o-NC, both miR-204 mimic and oe-NC, and both miR-204 mimic and oe-BRUCE. A The miR-204 expression and mRNA expression of BRUCE in cells detected by RTqPCR. B The protein expression of BRUCE in cells detected by Western blot analysis. C The fusion of autophagosome and lysosome in cells detected by immunofluorescence staining $(400 \times)$. D The immunofluorescence staining of axons in HT22 cells detected by immunofluorescence staining. ${ }^{*} p<$ 0.05 compared with cells treated with both mimic-NC ad oe-NC; ${ }^{p} p<0.05$ compared with cells treated with both miR-204 mimic and oe-NC. The experiment was repeated three times independently.

of miR-204 mimic and oe-BRUCE led to significantly enhanced autophagosome-lysosome fusion (Fig. 6C). Furthermore, results from the morphological detection for the axons of HT22 cells showed that the axon length and size of cell body were reduced in cells treated with both miR-204 mimic and oe-NC, but showed an opposite result in cells treated with both miR-204 mimic and oeBRUCE (Fig. 6D and Fig. S1E). According to the above results, the promoting effect of miR-204 on AD axonal dystrophy can be eliminated by BRUCE overexpression.

\section{Discussion}

Axonal dystrophy, as one of the early pathological characteristics of neurodegenerative diseases including $\mathrm{AD}$, is potentially causative in vesicular trafficking impairment, neuronal damage, and/or mortality ${ }^{18}$. It has been reported in a former study that the microtubule-stabilizing agent, Epothilone D could alleviate the disorders including axonal dysfunction and AD-like pathology in aged Tau transgenic mice ${ }^{19}$. However, the specific mechanism underlying the dystrophic axons in AD is still not well-characterized, thus more investigations are required to develop effective therapies for AD treatment. Most importantly, it has been demonstrated that functional-impaired crucial secretory peptidic cargos including BDNF were involved in the development of $\mathrm{AD}^{20}$. STX17 was evolutionarily conserved in the fusion of autophagosome and lysosomes ${ }^{21}$, while BRUCE was reported to participate in the regulation of autophagosome-lysosome fusion $^{22}$. miR-204 was also reported to be involved in the axonal guidance where the abnormal expression of miR-204 was observed in mesial temporal lobe epilepsy ${ }^{23}$. Based on these findings, we hypothesized that the BDNF/BRUCE/STX17/miR-204 axis affects the development of axonal dystrophy in $\mathrm{AD}$ via regulation of autophagosome-lysosome fusion. Eventually, the findings from the present study demonstrated that silencing of miR-204 contributed to the promotion of BRUCE, and resulted in enhanced STX17-mediated autophagosome-lysosome fusion, thus improving the BDNF nuclear transport in neurons. 
The results obtained from the present study revealed that the intranuclear expression of BDNF and the expression of BDNF/TrkB signaling pathway-related factors ( $p-\operatorname{Trk} B / \operatorname{Trk} B$ and $p-C R E B / C R E B)$ were reduced in the hippocampus brain area of $\mathrm{AD}$ mice, accompanied by swollen and shortened axons. A former study has reported that BDNF and TrkB-TK+ were downregulated in the hippocampus of patients suffering from schizophrenia and mood disorders ${ }^{24}$, which was consistent with our results that APP/PS1 transgenic mice present BDNF/TrkB signaling deficits. The pronounced decline in the levels of $\mathrm{BDNF}$ and TrkB was also found in the early stage of $\mathrm{AD}^{25}$. Additionally, reduced expression of BDNF exons IX and IV, and decreased CRE (BDNF) binding activity was observed in the hippocampus area of glucocorticoid receptor impaired mice ${ }^{26}$. Furthermore, based on the definition of axonal dystrophy, swollen and tortuous neuronal axons have induced synaptic changes in $\mathrm{AD}^{27}$. Therefore, the previous findings were consistent with the results of the present study, where the poorly expressed BDNF/TrkB signaling pathway was correlated with axonal dystrophy in $\mathrm{AD}$.

In addition, results from the present study revealed that the induction of axonal dystrophy by repressing the autophagosome-lysosome fusion has reduced the BDNF nuclear transport. As mentioned above, autophagosome was the key component in dystrophic axons, and lysosomes were the crucial component involved in the neuronal function and mutations in genes inducing lysosome formation, as well as activity related to neurodegenerative disorders ${ }^{10,28}$. A previous study has been revealed that the retrograde transport of BDNF-activated TrkB receptors participated in the process of autophagosomes to promote neuronal complexity and prevented neurodegeneration in vivo ${ }^{8}$. The present study demonstrated that BRUCE interacted with STX17, and the silencing of both BRUCE and STX17 could reduce the autophagosome-lysosome fusion. Similarly, the interaction between BRUCE and STX17 was also proven in former research, where BRUCE modulated the STX17mediated autophagosome-lysosome fusion ${ }^{11}$. Furthermore, this study revealed the upregulation of miR-204 in hippocampus tissues of AD mice as well as in HT22 cells and showed that miR-204 targeted BRUCE, thus resulting in suppressed autophagosome-lysosome fusion and dystrophic axons. However, the recovered expression of BRUCE has reversed all the effects induced by miR-204. The highly expressed miR-204 in the distal axons of sympathetic neurons has also been identified in a previous study ${ }^{13}$. Downregulation of miR-204 which contributed to the elevated expression of LC3II, a key biomarker of the autophagosome, in myocardial ischemia-reperfusion injury has also been reported in a previous research ${ }^{29}$. Most importantly, BRUCE enhanced

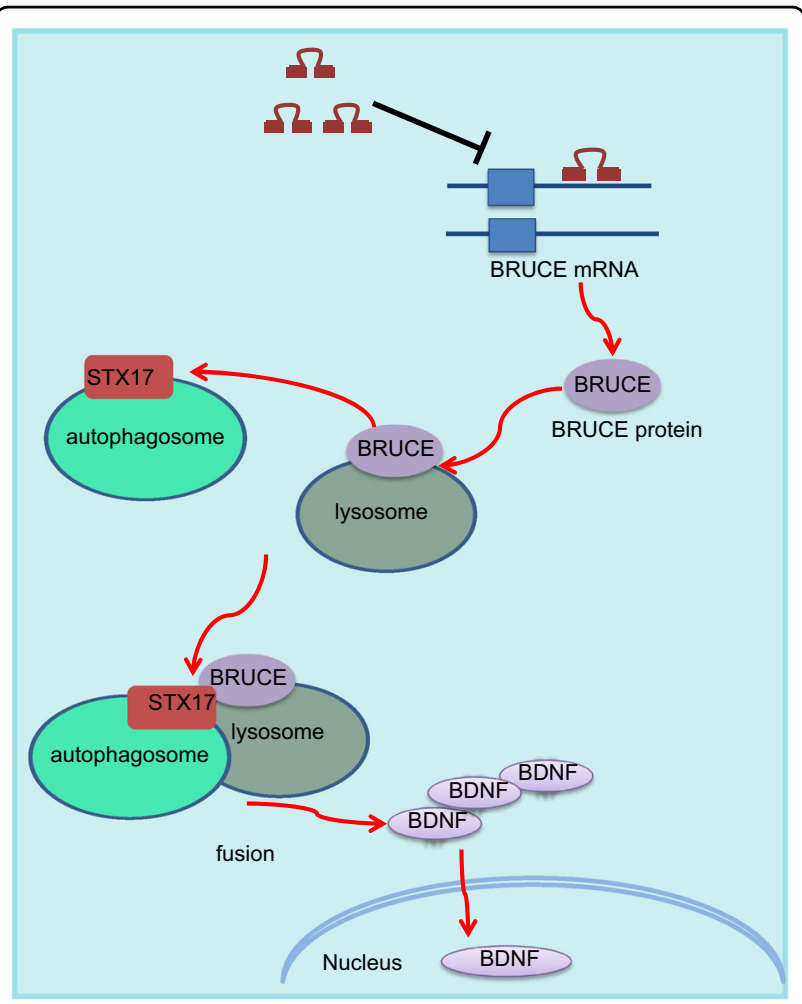

Fig. 7 The regulatory mechanism involving miR-204/BRUCE/ STX17/BDNF that affects the dystrophic axons induced by $A D$ via autophagosome-lysosome fusion mediation. Silencing of miR-204 promoted BRUCE to enhance the STX17-mediated autophagosomelysosome fusion, thus improving the BDNF nuclear transport and alleviating the dystrophic axons of AD mice.

the fusion of autophagosome and lysosome ${ }^{11}$. This evidence is in line with our study where the role of silenced miR-204 and overexpressed BRUCE which contributed to the promotion of autophagosome-lysosome fusion and amelioration of axonal dystrophy in AD was proven.

In conclusion, the key findings of the present study suggest inhibition of miR-204 prevents defective axonal transport by blocking BRUCE interaction with STX17 in hippocampal neurons. This study provides new insight into novel therapeutic targets for AD treatment (Fig. 7). Nevertheless, it should be also pointed out that more in-depth investigations are still required to explore the underlying mechanism of BDNF mediating autophagosome-lysosome fusion. It is also necessary to further identify the specific interaction between miR204 and IAP family members since their targeting relationship has been rarely reported.

\section{Acknowledgements}

We acknowledge and appreciate our colleagues for their valuable efforts and comments on this paper. This work was supported by the Natural Science Foundation of China (No. 81871033). 


\section{Author contributions}

Lu Zhang and Yu Fang designed the study, Xinyu Zhao participated in the data collection, Yake Zheng and Yunqing Ma performed the statistical analysis. Shuang Li prepared figures. Zhi Huang and Lihao Li drafted the paper. All authors read and approved the final paper.

\section{Conflict of interest}

The authors declare no competing interests.

\section{Publisher's note}

Springer Nature remains neutral with regard to jurisdictional claims in published maps and institutional affiliations.

Supplementary information The online version contains supplementary material available at https://doi.org/10.1038/s41398-021-01427-2.

Received: 30 November 2020 Revised: 24 April 2021 Accepted: 6 May 2021 Published online: 05 August 2021

\section{References}

1. Stokin, G. B. et al. Axonopathy and transport deficits early in the pathogenesis of Alzheimer's disease. Science 307, 1282-1288 (2005).

2. Beard, H. et al. Axonal dystrophy in the brain of mice with Sanfilippo syndrome. Exp. Neurol. 295, 243-255 (2017).

3. Nardocci, N. \& Zorzi, G. Axonal dystrophies. Handb. Clin. Neurol. 113, 1919-1924 (2013).

4. Molla, B. et al. Phosphodiesterase inhibitors revert axonal dystrophy in Friedreich's ataxia mouse model. Neurotherapeutics 16, 432-449 (2019).

5. Zhang, B. et al. A brain-penetrant triazolopyrimidine enhances microtubulestability, reduces axonal dysfunction and decreases tau pathology in a mouse tauopathy model. Mol. Neurodegener. 13, 59 (2018).

6. McGregor, C. E. \& English, A. W. The role of BDNF in peripheral nerve regeneration: activity-dependent treatments and Val66Met. Front. Cell Neurosci. 12, 522 (2018).

7. Savolainen, M., Emerich, D. \& Kordower, J. H. Disease modification through trophic factor delivery. Methods Mol. Biol. 1780, 525-547 (2018).

8. Kononenko, N. L. et al. Retrograde transport of TrkB-containing autophagosomes via the adaptor AP-2 mediates neuronal complexity and prevents neurodegeneration. Nat. Commun. 8, 14819 (2017).

9. Evans, S. F. et al. Neuronal brain-derived neurotrophic factor is synthesized in excess, with levels regulated by sortilin-mediated trafficking and lysosomal degradation. J. Biol. Chem. 286, 29556-29567 (2011).

10. Snouwaert, J. N. et al. A mutation in the Borcs7 subunit of the lysosome regulatory $B O R C$ complex results in motor deficits and dystrophic axonopathy in mice. Cell Rep. 24, 1254-1265 (2018).

11. Ebner, P. et al. The IAP family member BRUCE regulates autophagosomelysosome fusion. Nat. Commun. 9, 599 (2018).

12. Uematsu, M., Nishimura, T., Sakamaki, Y., Yamamoto, H. \& Mizushima, N. Accumulation of undegraded autophagosomes by expression of dominantnegative STX17 (syntaxin 17) mutants. Autophagy 13, 1452-1464 (2017).
13. Natera-Naranjo, O., Aschrafi, A., Gioio, A. E. \& Kaplan, B. B. Identification and quantitative analyses of microRNAs located in the distal axons of sympathetic neurons. RNA 16, 1516-1529 (2010).

14. Conte, I. et al. The combination of transcriptomics and informatics identifies pathways targeted by miR-204 during neurogenesis and axon guidance. Nucleic Acids Res. 42, 7793-7806 (2014).

15. Imam, J. S. et al. Genomic loss of tumor suppressor miRNA-204 promotes cancer cell migration and invasion by activating AKT/mTOR/Rac1 signaling and actin reorganization. PLoS One 7, e52397 (2012).

16. Chi, C. et al. LAMP-2B regulates human cardiomyocyte function by mediating autophagosome-lysosome fusion. Proc. Natl Acad. Sci. USA 116, 556-565 (2019).

17. Mauvezin, C., Nagy, P., Juhasz, G. \& Neufeld, T. P. Autophagosome-lysosome fusion is independent of V-ATPase-mediated acidification. Nat. Commun. $\mathbf{6}$, 7007 (2015).

18. Ovsepian, S. V., O'Leary, V. B., Zaborszky, L., Ntziachristos, V. \& Dolly, J. O. Amyloid plaques of Alzheimer's disease as hotspots of glutamatergic activity. Neuroscientist 25, 288-297 (2019).

19. Zhang, B. et al. The microtubule-stabilizing agent, epothilone D, reduces axonal dysfunction, neurotoxicity, cognitive deficits, and Alzheimer-like pathology in an interventional study with aged tau transgenic mice. J. Neurosci. 32, 3601-3611 (2012).

20. $\mathrm{Pla}, \mathrm{V}$. et al. Secretory sorting receptors carboxypeptidase $\mathrm{E}$ and secretogranin III in amyloid beta-associated neural degeneration in Alzheimer's disease. Brain Pathol. 23, 274-284 (2013)

21. Hegedus, K., Takats, S., Kovacs, A. L. \& Juhasz, G. Evolutionarily conserved role and physiological relevance of a STX17/Syx17 (syntaxin 17)-containing SNARE complex in autophagosome fusion with endosomes and lysosomes. Autophagy 9, 1642-1646 (2013).

22. Ikeda, F. The anti-apoptotic ubiquitin conjugating enzyme BIRC6/BRUCE regulates autophagosome-lysosome fusion. Autophagy 14, 1283-1284 (2018).

23. Kaalund, S. S. et al. Aberrant expression of miR-218 and miR-204 in human mesial temporal lobe epilepsy and hippocampal sclerosis-convergence on axonal guidance. Epilepsia 55, 2017-2027 (2014).

24. Thompson Ray, M., Weickert, C. S., Wyatt, E. \& Webster, M. J. Decreased BDNF, trkB-TK+ and GAD67 mRNA expression in the hippocampus of individuals with schizophrenia and mood disorders. J. Psychiatry Neurosci. 36, 195-203 (2011).

25. Devi, L. \& Ohno, M. TrkB reduction exacerbates Alzheimer's disease-like signaling aberrations and memory deficits without affecting beta-amyloidosis in 5XFAD mice. Transl. Psychiatry 5, e562 (2015).

26. Alboni, S. et al. Stress induces altered CRE/CREB pathway activity and BDNF expression in the hippocampus of glucocorticoid receptor-impaired mice Neuropharmacology 60, 1337-1346 (2011).

27. Blazquez-Llorca, L. et al. High plasticity of axonal pathology in Alzheimer's disease mouse models. Acta Neuropathol. Commun. 5, 14 (2017).

28. Liberski, P. P. Axonal changes in experimental prion diseases recapitulate those following constriction of postganglionic branches of the superior cervical ganglion: a comparison 40 years later. Prion 13, 83-93 (2019).

29. Xiao, J. et al. MiR-204 regulates cardiomyocyte autophagy induced by ischemia-reperfusion through LC3-II. J. Biomed. Sci. 18, 35 (2011). 